\title{
Factores Influyentes en Motivación y Estrategias de Aprendizaje en los Alumnos de Grado
}

\section{Influential Factors in Motivation and Learning Strategies in Grade Students}

\author{
Daniel Garrote Rojas * \\ Cristina Garrote Rojas \\ Sara Jiménez Fernández \\ Universidad de Castilla-La Mancha
}

\begin{abstract}
Analizando los diferentes procesos mentales que se dan durante el aprendizaje desde diferentes paradigmas, donde el docente pasa de ser el protagonista y responsable del aprendizaje del alumno a ser quien planifica la enseñanza y la organiza para que sus alumnos realicen un aprendizaje significativo. En el aprendizaje autorregulado los aprendices son los que eligen y deciden sobre su conducta, siendo los artífices y promotores de sus aprendizajes. A través de este estudio sobre una muestra de estudiantes universitarios, utilizando el instrumento CEAM II (Cuestionario de Estrategias de Aprendizaje y Motivación), formado por preguntas sobre la motivación y las estrategias de aprendizaje pretendemos conocer la implicaciones de las diferentes dimensiones que lo forman. El análisis de las diferentes variables nos lleva a conocer qué valores son los más empleados por los estudiantes dentro de los factores de motivación (metas intrínsecas, valor de la tarea y autoeficacia) y de las estrategias de aprendizaje (elaboración, organización y metacognición), así como aquellos ítems más y menos valorados por los mismos. Los docentes debemos trasmitir a los estudiantes los contenidos de las diferentes asignaturas a través de una metodología que les resulte atractiva, así les facilitaremos la adquisición de los mismos.
\end{abstract}

Descriptores: Estrategias de aprendizaje, Motivación, Rendimiento, Evaluación, CEAM II.

Analyzing the different mental processes that occur during learning from different paradigms, where the teacher happens to be the protagonist and responsible for student learning to be who plans and organizes teaching for their students make significant learning. It should motivate, highlighting the importance of the matter and its implications for acredentar interest in it. Self-regulated learning learners are those who choose and decide on their conduct, being the architects and promoters of their learning.. Through this study on a sample of university students using the CEAM II (Learning Strategies Questionnaire and Motivation) instrument, consisting of questions about motivation and learning strategies we tried to understand the implications of different dimensions that form. The analysis of the different variables leads us to know which values are the most used by students in motivational factors (intrinsic goals, task value and self-efficacy) and learning strategies (preparation, organization and metacognition) as well as those items most and least valued by them. Teachers must convey to students the contents of different subjects through a methodology that is attractive to them, so we will provide the acquisition thereof.

Keywords: Learning strategies, Motivation, Performance, Assessment, CEAM II.

*Contacto: daniel.garrote@uclm.es

ISSN: $1696-4713$

www.rinace.net/reice/
Recibido: 21 de noviembre 2014

$1^{\text {a }}$ Evaluación: 13 de febrero 2015

$2^{\text {a }}$ Evaluación: 19 de junio 2015

Aceptado: $\quad 20$ de agosto 2015 


\section{Introducción}

La Universidad, como institución creadora y transmisora de ciencia, técnica y cultura, tiene la responsabilidad, no sólo de preparar para el ejercicio de las actividades profesionales al alumno, sino que tiene un compromiso de excelencia para la docencia, la investigación y la transferencia. Para poder ofrecer una buena formación, es necesario que el profesorado tenga en cuenta las características de aprendizaje del alumnado de forma que la acción docente considere esta premisa con el fin de aunar estrategias de enseñanza y aprendizaje.

El Conductismo y la Psicología Cognitiva son las responsables de la explicación del aprendizaje. El paradigma conductista postula que la explicación de la conducta humana se da a través de estímulos y respuestas (Bara, 2001; González-Pienda, González, Núñez y Valle, 2002), siendo el aprendizaje una conexión entre estímulos y respuestas (Bernad, 2000). Avalando esta afirmación a través de dos teorías: el condicionamiento clásico y el condicionamiento operante. En la primera, el aprendizaje se produce por una asociación entre estímulos y respuestas, mientras que en la segunda, se considera el reforzamiento un elemento fundamental que hace posible que haya una respuesta frente a un estímulo. Así según Bara (2001) el alumno durante el aprendizaje tiene una función pasiva ya que es un simple receptor de contenido a través de un emisor, que es el docente, quien tiene toda la responsabilidad en el proceso de aprendizaje. Siendo únicamente relevantes los resultados del alumno.

El paradigma cognitivo, por el contrario frente al conductismo, considera al alumno un sujeto activo en el proceso de aprendizaje (Sampascual, 2001). Considera que los procesos mentales que se dan dentro del alumno son fundamentales para comprender la conducta humana y el aprendizaje. La teoría del Procesamiento de la Información (Bara, 2001) fue la que desgaja el vínculo entre el estímulo y la respuesta, desplazando el interés a los procesos mentales humanos (Delclaux, 1982), donde se considera que algunas operaciones simbólicas como codificar, comparar, localizar, etc., pueden influir en crear conocimiento (Lachman, Lachman y Butterfield, 1979). En el interior del sujeto se da el pensamiento, razonamiento, memorización, codificación, organización, elaboración, etc., de toda la información que recibe y con los conocimientos que ya posee se da en él un aprendizaje comprensivo.

Las estrategias de aprendizaje siguen constituyendo una de las áreas cruciales en la evaluación e intervención psicoeducativa como ya decía Vizcarro en 1992 y avaló el informe de la OCDE (Organización para la Cooperación y Desarrollo Económico) en 1989 donde aumentaban las investigaciones sobre habilidades y estrategias de aprendizaje (Monereo, 2000).

\section{Fundamentación Teórica}

Es a partir de 1960 cuando se dejan atrás los modelos de aprendizaje conocidos como "de caja negra”, donde se daba importancia a las variables de entrada y salida, es decir, la enseñanza y el rendimiento. Sin tenerse en cuenta los procesos que se dan en el sujeto (Shulman, 1986).

Las teorías cognitivas de entonces se centran en el alumno como procesador activo, modificando la información que toma del medio (Pozo, 1989). Comenzando a 
considerarse que es más rentable hacer hincapié en la mejora de los procesos y estructuras de pensamiento que hacerlo en las variables externas al sujeto: materiales y métodos instructivos (Chipman y Segal, 1985). De forma paralela a estas conductas cognitivas, aparece un interés relacionado con el aprendizaje y el rendimiento de procesos internos como son la afectividad y la motivación.

A lo largo de los años 60 y 70, ambas líneas de investigación, cognitiva y motivacional han avanzado por caminos diferentes, pero en los años 80 aparecen nuevas corrientes que pretenden unir ambos componentes para mejorar el aprendizaje y el rendimiento (González y Tourón, 1992). Considerando la teoría del aprendizaje de Bloom (1976) como base de este nuevo camino debido a que considera tres factores fundamentales en los resultados del aprendizaje: la calidad de enseñanza, las conductas cognitivas del alumno y las características afectivas del estudiante. Este cambio en el estudio del aprendizaje donde se profundiza en los procesos mentales superiores (Delclaux, 1982; Gagné, 1991; De Vega, 1989) como la atención, la memoria, el pensamiento y el lenguaje, que no son observables (Bara, 2001). Esta visión implica en el discente, un proceso de adquisición y reorganización de las estructuras cognitivas de manera constante (Sampascual, 2001).

Este cambio de paradigma, del conductista al cognitivista se da en tres etapas: aprendizaje como adquisición de respuestas, aprendizaje como adquisición de conocimientos y aprendizaje como constructo de conocimientos. Lo que da lugar a dos teorías, el Procesamiento de la Información y el Constructivismo.

La teoría del Procesamiento de la Información propone una concepción del aprendizaje con carácter cognitivo, donde se consideran los procesos mentales internos el objeto de estudio para la comprensión del aprendizaje (Pozo, 1989). Considerando que operaciones simbólicas básicas como codificar, comparar, localizar o almacenar pueden dar lugar a la inteligencia humana, así como mostrar la capacidad de crear conocimiento, innovaciones y expectativas en el futuro (Lachman, Lachman y Butterfield, 1979). De forma que los eventos mentalmente informacionales, conscientes o inconscientes, están formados por un imput de información, una información que se ejecuta al respecto y un output o resultado de información que llegó al sistema (Palmer y Kimchi, 1986, citados por Ballesteros, 1997). Cumpliéndose así el supuesto de la descomposición recursiva de los procesos cognitivos. En el que la mente funcionaría como un ordenador en el que los procesos que ejecuta se podrían desglosar en unidades mínimas que lo forman (Pozo, 1989). Se podría describir el procesamiento de la información con la siguiente secuencia (Carretero, 2001; Gargallo, 2006; Rodríguez Neira, 1999; Sampascual, 2001):

- A través de los registros sensoriales, receptores o memoria sensorial (que es limitada), se capta la información.

- La información es almacenada en la memoria a corto plazo o memoria de trabajo, con capacidad limitada cuya función es la de almacenamiento temporal previo a que la información pase a la memoria a largo plazo.

- Por último, la información pasa de la memoria a largo plazo desde la memoria a corto plazo cuya capacidad de almacenaje es ilimitada.

A raíz de la teoría del Procesamiento de la Información, se ve la necesidad de enseñar a los alumnos explícitamente en habilidades cognitivas y metacognitivas. Para que obtengan autonomía en sus procesos de aprendizaje. Como defienden los teóricos del 
Procesamiento de la Información: "Todo proceso puede reducirse y simplificarse en una serie subprocesos o procesos de nivel inferior y más simple"; "Cualquier proceso o ejecución cognitiva puede ser comprendida reduciéndola a las unidades mínimas de que está compuesta" (Pozo, 1989, p. 45). Así, cualquier tarea de aprendizaje puede desglosarse en sub-tareas que hagan más sencilla la obtención del objetivo por parte del alumno. Por tanto, el alumno pasa de reproductor de la información (teoría conductista) a sujeto activo en su aprendizaje a través del procesamiento de la información que dependen de los procesos internos que lleva a cabo. Debiéndose utilizar metodologías activas y participativas donde el protagonista sea el alumno en vez del profesor. Hernández Rojas (1998) indica que en el diseño desde una institución del nivel educativo que se le exigirá a los alumnos hay que conocer la base desde la que parten conocimiento previo, desarrollo cognitivo, conocimiento estratégico, así como las expectativas y motivos para así programas los objetivos dirigidos a promover su aprendizaje.

En este proceso de enseñanza y aprendizaje el docente pasa de ser el protagonista y responsable del aprendizaje del alumno (teoría conductista) a ser este quien planifica la enseñanza y la organiza para que sus alumnos realicen un aprendizaje significativo. Para ello, el docente también debe motivar al alumnado, resaltando la relevancia de la materia y su repercusión para que así traslade al discente el interés por la materia.

No obstante, la teoría del Procesamiento de la Información presenta algunas limitaciones. En relación al análisis de los procesos internos, este no puede llevarse a cabo en su plenitud, ya que la conciencia, está considerada con una concepción pasiva. Se analizan otros conceptos como la memoria a corto plazo, la atención selectiva, etc.

Pozo (1989) considera que a través de la teoría del Procesamiento de la Información, cualquier proceso o ejecución cognitiva puede entenderse reduciéndolo a las unidades mínimas de las que está compuesta. No se considera la dimensión afectiva, emocional y/o sentimental, aunque influyen en el aprendizaje. Se realiza una escasa consideración (Gargallo, 2006; Soler y Alonso, 1996). Sin embargo, hay un gran interés por los estilos, enfoques y estrategias de aprendizaje en el procesamiento de información.

Algunas limitaciones serán atendidas por la teoría constructivista (Gargallo, 2006). En el Constructivismo el conocimiento es un proceso activo del ser humano (Aznar, 1992; Carretero, 1993) donde se adquieren los conocimientos a través de un proceso de transformación, interpretación e integración en sus estructuras de conocimiento ya disponibles (Gómes y Gargallo, 1993) en el que los aspectos emotivo-afectivos y semánticos son importantes en la significación de los aprendizajes. Piaget, Vygotsky, Bruner y Ausubel han realizado las aportaciones más importantes dentro de la teoría constructivista. Piaget, en la teoría de la equilibración, el proceso cognitivo del estudiante se da mediante un proceso de equilibración entre el proceso de asimilación y el de acomodación. El primero consiste en la adquisición de la información del medio por parte del organismo. Esta información se reintegra e interpreta en base a los esquemas y estructuras ya existentes en el organismo. Tendiendo a incorporar los elementos exteriores a él que son compatibles con su naturales (Piaget, 1978). En el proceso de acomodación se modifican los esquemas ya existentes en base a la nueva información recibida. Es un proceso de ajuste del organismo a las condiciones cambiantes del medio que permiten simular nuevas situaciones al modificar las pautas de comportamiento previas (Garfella y Gargallo, 1993; Piaget, 1978). La superación de este conflicto cognitivo (desequilibrio entre ambos procesos) produce la reconstrucción del 
conocimiento. Entendiéndose que el docente debe provocar a sus alumnos continuos conflictos o disonancias cognitivas. Deben llevarse a cabo dentro de la "zona de desajuste óptimo", donde la nueva información recibida tenga la dificultad suficiente para que el alumno supere el conflicto cognitivo.

Vigotsky enfatiza en el contexto como medio para el desarrollo del aprendizaje. Así, este se da fuera del sujeto y debe evolucionar hasta que se logre su interiorización. Lo denominó, la ley de la doble formación de conceptos, a través de la cual, todos los conocimientos se adquieren dos veces, primero externamente y posteriormente se interioriza (Pozo, 1989). Diferencia dos conceptos en su entendimiento sobre el aprendizaje, el nivel de desarrollo actual y el nivel de desarrollo potencial (Pozo, 1989). El primero hace referencia a los ciclos evolutivos (Cánovas y Rius, 1993), se caracteriza por aquello que el alumno es capaz de llevar a cabo por sí solo, de forma autónoma y sin ayuda exterior. El nivel de desarrollo potencial se refiere a lo que el sujeto es capaz de conseguir con ayuda de otras personas o elementos mediadores. La diferencia entre ambos niveles forma la zona de desarrollo potencial (Pozo, 1989; Reig y Gradolí, 1992). En referencia a la educación, es de gran relevancia potenciar la acción de los mediadores en el desarrollo del aprendizaje. El objetivo de la educación debe ser trabajar la zona de desarrollo potencial, así, los docentes deben dar a los alumnos las herramientas necesarias para que estos progresen en sus aprendizajes.

Bruner considera, al igual que Vigotsky, que el aprendizaje es un proceso social (Bruner, 1991). A través de tres sistemas de representación de la realidad, tienen como objetivo el establecimiento de regularidades para formar categorías que permitan la integración del conocimiento en la estructura del sujeto, dando lugar al aprendizaje.

Ausubel, para lograr el aprendizaje significativo establece los conceptos inclusores, son entidades específicas en la estructura cognitiva que permiten el aprendizaje de nuevos elementos. Cuando no se produce una relación significativa aparecen los organizadores previos, son puentes cognitivos que se establecen entre los conceptos inclusores y los materiales nuevos a aprender. Así, el docente debe posibilitar la relación de aprendizajes significativos en los alumnos. Los materiales deben estar bien organizados para que se produzca el establecimiento de relaciones con el conocimiento previo del alumno. Se han de poner en práctica metodologías activas y participativas en las clases, que hagan al alumno reflexionar y el uso de conocimientos anteriormente aprendidos. La evaluación debe evitar la reproducción literal de los conceptos, sino llevarse a cabo mediante la reactivación, reflexión y puesta en práctica de los conocimientos adquiridos para poder resolver las tareas que se soliciten.

En el proceso de enseñanza aprendizaje influyen tanto los factores personales como los socio-ambientales y propios de cada estudiante, formándose un complejo sistema interactivo (Navaridas, 2002). El nivel con el que se involucran los padres en la educación de sus hijos influyen en la motivación y logros de este (Amstrong, 1991). Los maestros desde la escuela no tienen la exclusividad del aprendizaje, sino que los padres son una vía de ayuda para que estos aprendan en diferentes contextos (Gallego, 2008). El nivel educativo de los padres está relacionado con el rendimiento académico de los hijos. A mayor número de años de educación de los padres, mayor es el tiempo dedicado al estudiante, así como la calidad de la supervisión al mismo (Martínez et al., 2010). 


\section{Objetivos}

Los diferentes estudios que se han realizado sobre la influencia de los elementos afectivo-motivacionales sobre el funcionamiento cognitivo han perfilado instrumentos que miden aspectos relacionados tanto con lo cognitivo como con lo motivacional (Cardozo, 2008; Parrales y Solórzano, 2014; Roces, Tourón y González, 1995). Sin ser abundantes aquellos instrumentos que miden ambas dimensiones.

Para dar respuesta a cómo la motivación y las estrategias de aprendizaje influyen en el rendimiento académico, buscaremos:

- Conocer las actitudes y conductas motivacionales empleadas por parte de los alumnos.

- Describir las estrategias de aprendizaje utilizadas.

- Analizar las variables que más influyen en la motivación.

- Profundizar en las variables con mayor repercusión en las estrategias de aprendizaje.

\section{Metodología}

Para poder adentrarnos en aquellas situaciones que activan a los individuos para obtener las metas que desean en el mundo académico, vamos a emplear una metodología cuantitativa a través del uso del cuestionarios CEAM con el fin de conocer esta realidad en el Grado de Infantil y Primaria de la Facultad de Educación de Albacete.

\subsection{Muestra}

La muestra seleccionada la forman 621 alumnos, 239 de primer curso y 382 de cuarto curso del Grado de Maestro en Infantil y Primaria en la Facultad de Educación de Albacete de la Universidad de Castilla-La Mancha. El criterio por el que se utilizó la selección de la misma ha sido de contacto directo con la población activa estudiantil.

\subsection{Instrumento de evaluación}

El cuestionario utilizado ha sido el CEAM II, que proviene de la traducción y adaptación del MSLQ (Motivated Strategies for Learning Questionnaire), desarrollado por Pintrich y otros (1991), pretende formar una visión cognitiva de la motivación y de las estrategias de aprendizaje del alumnado. Este modelo conceptual de la motivación es una adaptación del modelo motivacional de expectativas y valores (Eccles, 1983; Pintrich, 1988). Para Pintrich y DeGroot (1990) se dan tres elementos motivacionales en las estrategias de aprendizaje: un componente de expectativas, un componente de valor y un componente afectivo. Respecto a las estrategias de aprendizaje consideran relevantes tres aspectos: las estrategias metacognitivas para planificar, dirigir y modificar el funcionamiento cognitivo, el control de recursos disponibles y las estrategias cognitivas que los sujetos emplean para entender, aprender y recordar la materia de estudio (Printrich y DeGroot, 1990).

El MSLQ se ha utilizado en el ámbito tanto universitario como no universitario, siendo sometida cada versión a diferentes análisis psicométricos de fiabilidad, análisis factorial y correlaciones con diferentes medidas de rendimiento académico. Respecto al trabajo 
realizado por Roces C., Tourón J. y González M.C. (1995) sobre la adaptación y traducción al castellano del cuestionario, han obtenido dos versiones del CEAM (Cuestionario de Estrategias de Aprendizaje y Motivación): CEAM I, motivación y estrategias de aprendizaje en una asignatura concreta y el CEAM II (tabla 1), motivación y estrategias que un alumno lleva a cabo en las asignaturas que forman un curso completo.

Tabla 1. Escalas y dimensiones del CEAM II

\begin{tabular}{|c|c|c|}
\hline ESCALAS & DIMENSIONES & SUB-ESCALAS \\
\hline \multirow{3}{*}{ Motivación } & Componentes de expectativas & $\begin{array}{l}\text { - Creencias de control } \\
\text { - Autoeficacia }\end{array}$ \\
\hline & Componentes de valor & $\begin{array}{l}\text { - Metas intrínsecas } \\
\text { - Metas extrínsecas } \\
\text { - Valor de la tarea }\end{array}$ \\
\hline & Componentes afectivos & - Ansiedad en los exámenes \\
\hline \multirow[t]{2}{*}{ Estrategias de aprendizaje } & $\begin{array}{l}\text { Estrategias cognitivas y } \\
\text { metacognitivas }\end{array}$ & $\begin{array}{l}\text { - Repetición } \\
\text { - Elaboración } \\
\text { - Organización } \\
\text { - Pensamiento crítico } \\
\text { - Meta-cognición }\end{array}$ \\
\hline & $\begin{array}{l}\text { Estrategias de manejo de } \\
\text { recursos }\end{array}$ & $\begin{array}{l}\text { - Tiempo y lugar de estudio } \\
\text { - Regulación del esfuerzo } \\
\text { - Aprendizaje con otros } \\
\text { - Búsqueda de ayuda }\end{array}$ \\
\hline
\end{tabular}

Fuente: Elaboración propia.

El CEAM II, consta de 81 ítems, 31 hacen referencia a la motivación y 50 a estrategias de aprendizaje. El tipo de respuesta es tipo Likert de 7 puntos (1: No me describe en absoluto, 2: Me describe un poco, 3: Me describe moderadamente, 4: No estoy seguro(a), 5: Me describe suficientemente, 6: Me describe mucho, 7: Me describe totalmente).

\section{Resultados}

A través del programa estadístico SPSS hemos obtenido los resultados. En primer lugar hemos llevado a cabo una clasificación de los factores motivacionales que componen el cuestionario en función del valor medio que se ha obtenido (tabla 2), conociendo así los aspectos más y menos valorados por parte de los alumnos. El factor motivacional que obtiene la media más elevada, las metas intrínsecas con 6,07. Este componente hace referencia a la percepción por parte del estudiante de las razones por las que se implica en una tarea de aprendizaje, es el grado en que el estudiante considera que toma parte en una tarea por razones como el reto, la curiosidad o el dominio. La puntuación más baja se obtiene en la ansiedad (3,11). Recoge los pensamientos negativos de los alumnos durante los exámenes, los cuales interfieren en los resultados y las reacciones fisiológicas a las que hacen frente los estudiantes durante la realización de la prueba. Se compone de los siguientes aspectos: nerviosismo, aceleración del pulso, pensamientos sobre lo mal que lo está haciendo, consecuencias de suspender la prueba o las preguntas que se desconocen la respuesta. Una ausencia de pensamientos ansiosos durante el examen permite concentrarse y rendir mejor.

En los valores medios, la distribución de mayor a menor sería: valor de la tarea, autoeficacia, metas extrínsecas y creencias de control. 
Tabla 2. Motivación. Valores medios de los factores del cuestionario

\begin{tabular}{lc}
\hline \multicolumn{1}{c}{ FACTORES } & VALOR MEDIO \\
\hline I Autoeficacia & 5,57 \\
II Ansiedad & 3,11 \\
III Valor de la tarea & 5,91 \\
IV Metas Extrínsecas & 5,39 \\
V Metas Intrínsecas & 6,07 \\
VI Creencias control & 5,27 \\
\hline
\end{tabular}

Fuente: Elaboración propia.

En las estrategias de aprendizaje, como se observa en la tabla 3, la más utilizada por los estudiantes es la elaboración con una puntuación media de 5,30. La cual permite valorar si el alumno aplica conocimientos anteriores a nuevas situaciones para resolver problemas, tomar decisiones, hacer evaluaciones críticas y, si logra establecer conexiones entre la información nueva y la que ya poseía para poder recordarla mejor. La menos empleada es la autointerrogación $(4,45)$, en la que se tienen en cuenta las preguntas que se hace así mismo el estudiante con el objetivo de centrarse en el contenido de la materia, autoevaluar su nivel de comprensión y lograr cuestionar la veracidad de lo estudiado.

En los valores intermedios, por puntuaciones decrecientes, nos encontramos: organización, metacognición, ayuda y concentración.

Tabla 3. Estrategias de aprendizaje. Valores medios de los factores del cuestionario

\begin{tabular}{lc}
\hline \multicolumn{1}{c}{ FACTORES } & VALOR MEDIO \\
\hline VII Elaboración & 5,30 \\
VIII Concentración & 4,51 \\
IX Ayuda & 4,68 \\
X Organización & 5,17 \\
XI Autointerrogación & 4,45 \\
XII Metacognición & 5,06 \\
\hline
\end{tabular}

Fuente: Elaboración propia.

Analizando los aspectos motivacionales y las estrategias de aprendizaje con puntuaciones mayores y menores, hemos incluido cinco ítems dentro de cada uno. En la tabla 4 mostramos aquellos ítems que han obtenido mayor y menor puntuación en los aspectos motivacionales. Los ítems más valorados por la muestra han sido el ítem 21: "Confió en que puedo hacer un excelente trabajo respecto a las tareas". Pertenece a la orientación a metas extrínsecas, tener una alta orientación en esta escala hace que el estudiante se involucre en las tareas académicas para conseguir unos objetivos extrínsecos a la propia naturaleza de la tarea. En el ítem 26: "Me gusta esta asignatura". Pertenece a la orientación a metas intrínsecas, razones por las que se implica en la asignatura. El ítem 12: "Confío en que puedo aprender los conceptos básicos enseñados en esta asignatura”. Pertenece al factor autoeficacia para el rendimiento, las creencias que tienen los estudiantes sobre su propia capacidad para obtener un buen rendimiento académico. El ítem 17: "Estoy muy interesado en el contenido de esta asignatura". Pertenece al factor de motivaciones intrínsecas. El quinto ítem más valorado sería el ítem 18: "Si me esfuerzo lo suficiente, entenderé el contenido de la asignatura". Pertenece al factor de creencias de control y autoeficacia para el aprendizaje, una puntuación alta hace referencia a que el estudiante considera esta asignatura importante 
para su formación y útil para entender otras asignaturas e interesantes para lograr ambos objetivos.

Teniendo en cuenta los ítems menos valorados, cuatro de ellos hacen referencia a la ansiedad, ítem 19, 28, 3 y 14. Lo que indica que los estudiantes no se bloquean durante la realización de los exámenes con ideas negativas acerca de lo mal que lo están haciendo. Así como el ítem 25 perteneciente al factor de creencias de control y autoeficacia para el aprendizaje (tabla 4).

Tabla 4. Ítems de motivación más y menos valorados

\begin{tabular}{|c|c|c|}
\hline FACTOR & $\mathbf{N}^{\circ}$ & İTEMS MÁS VALORADO \\
\hline Motivación extrínseca & 21 & $\begin{array}{l}\text { Confío en que puedo hacer un excelente trabajo respecto a las } \\
\text { tareas y exámenes en esta asignatura. }\end{array}$ \\
\hline Motivación intrínseca & 26 & Me gusta esta asignatura. \\
\hline Rendimiento & 12 & $\begin{array}{l}\text { Confío en que puedo aprender los conceptos básicos enseñados en } \\
\text { esta asignatura. }\end{array}$ \\
\hline Motivación intrínseca & 17 & Estoy muy interesado en el contenido de esta asignatura. \\
\hline Creencias de control & 18 & $\begin{array}{l}\text { Si me esfuerzo lo suficiente, entenderé el contenido de la } \\
\text { asignatura. }\end{array}$ \\
\hline FACTOR & $\mathbf{N}^{\circ}$ & ÍTEMS MENOS VALORADO \\
\hline Ansiedad & 19 & $\begin{array}{l}\text { En esta asignatura, experimento una situación desagradable como } \\
\text { de "angustia". }\end{array}$ \\
\hline Ansiedad & 28 & $\begin{array}{l}\text { Siento angustia cuando presento un examen o tarea en esta } \\
\text { asignatura. }\end{array}$ \\
\hline Ansiedad & 3 & $\begin{array}{l}\text { Cuando presento una tarea, examen u otra actividad en esta } \\
\text { asignatura pienso que mi desempeño es deficiente comparado con } \\
\text { el de mis compañeros. }\end{array}$ \\
\hline Ansiedad & 14 & $\begin{array}{l}\text { Cuando presento una tarea, examen o actividad en esta asignatura } \\
\text { pienso en las consecuencias de un fracaso. }\end{array}$ \\
\hline Creencias de control & 25 & $\begin{array}{l}\text { Si no entiendo el contenido de la asignatura es porque no me } \\
\text { esfuerzo lo necesario. }\end{array}$ \\
\hline
\end{tabular}

Fuente: Elaboración propia.

Respecto a las estrategias de aprendizaje, en la tabla 5 podemos observar los ítems más y menos valorados. Respecto a los más valorados, el ítem 73: Asisto a clases regularmente. Hace referencia al factor constancia, la diligencia y el esfuerzo para llevar al día las actividades y trabajos de la asignatura para alcanzar las metas propuestas. El ítem 35: Usualmente estudio en un lugar donde pueda concentrarme. Pertenece al factor aprovechamiento del tiempo y concentración, el aprovechamiento que hace el estudiante de su tiempo de estudio y su capacidad para centrarse en aquella tarea que está realizando. Ítem 41: Cuando estoy confundido acerca de algo que estoy leyendo para esta asignatura, vuelvo a leerlo y trato de entenderlo. Se engloba en el factor metacognición, dirigida a reflejar la autorregulación metacognitiva, establecimiento de metas, regulación del estudio y de la propia comprensión. Ítem 63: Cuando estudio para esta asignatura, voy a mis apuntes y subrayo los conceptos importantes. Se encuentra dentro del factor de organización, aquellas estrategias que emplea el alumno para estudiar el contenido y seleccionar la información como esquemas, apuntes, artículos, etc. En un estudio realizado por Tourón (1989) sobre hábitos de estudio en universitarios, encontramos también como hábitos de estudio muy valorados los aspectos organizativos. El ítem 64: Cuando leo para esta asignatura trato de relacionar el material con el que ya conozco. Se encuentra dentro del factor de elaboración, permitiendo valorar si el alumno 
aplica conocimientos anteriores a situaciones nuevas para poder resolver problemas y establecer conexiones entre información nueva y la que ya poseía.

En relación a los ítems menos valorados, hacen referencia los que han obtenido menos valoración, ítem 37: "Frecuentemente me siento tan perezoso o aburrido cuando estudio para esta asignatura que abandono el estudio antes de finalizar lo que planeaba hacer". Lo cual hace relación al interés real que despierte la asignatura en estos estudiantes, sin ser una carga para ellos. Y el ítem 60: "Cuando el material y/o las tareas son difíciles, las abandono y sólo estudio las partes fáciles”. No considerando difíciles las tareas que deben realizar para superar la asignatura. Pertenecientes al factor constancia. El ítem 57: "Frecuentemente me doy cuenta de que no he comprendido bien lo que he leído para esta asignatura". Se encuentra dentro del factor metacognición. Los siguientes dos ítems pertenecen al factor aprovechamiento del tiempo y concentración. Ítem 33: "Durante las clase con frecuencia se me escapan puntos importantes, porque estoy pensando en otras cosas". Ítem 77: "Frecuentemente me percato de que no dedico mucho tiempo a esta asignatura debido a otras actividades". Debido a la forma en la que se imparte la asignatura y cómo se realizan las actividades, la falta de tiempo no es un aspecto destacado.

Tabla 5. Ítems de estrategias más y menos valorados

\begin{tabular}{|c|c|c|}
\hline$\overline{\text { FACTOR }}$ & $\mathrm{N}^{\mathbf{2}}$ & ÍTEMS MÁS VALORADO \\
\hline Constancia & 73 & Asisto a clases regularmente. \\
\hline Aprovechamiento & 35 & Usualmente estudio en un lugar donde pueda concentrarme. \\
\hline Metacognición & 41 & $\begin{array}{l}\text { Cuando estoy confundido (a) acerca de algo que estoy leyendo para esta } \\
\text { asignatura, vuelvo a leerlo y trato de entenderlo. }\end{array}$ \\
\hline Organización & 63 & $\begin{array}{l}\text { Cuando estudio para esta asignatura, voy a mis apuntes y subrayo los } \\
\text { conceptos importantes. }\end{array}$ \\
\hline Elaboración & 64 & $\begin{array}{l}\text { Cuando leo para esta asignatura trato de relacionar el material con el } \\
\text { que ya conozco. }\end{array}$ \\
\hline FACTOR & $\mathbf{N}^{\circ}$ & ÍTEMS MENOS VALORADO \\
\hline Constancia & 37 & $\begin{array}{l}\text { Frecuentemente me siento tan perezoso (a) o aburrido (a) cuando } \\
\text { estudio para esta asignatura que abandono el estudio antes de finalizar } \\
\text { lo que planeaba hacer. }\end{array}$ \\
\hline Constancia & 60 & $\begin{array}{l}\text { Cuando el material y/o las tareas son difíciles, las abandono y sólo } \\
\text { estudio las partes fáciles. }\end{array}$ \\
\hline Metacognición & 57 & $\begin{array}{l}\text { Frecuentemente me doy cuenta de que no he comprendido bien lo que } \\
\text { he leído para esta asignatura. }\end{array}$ \\
\hline Aprovechamiento & 33 & $\begin{array}{l}\text { Durante las clases con frecuencia se me escapan puntos importantes, } \\
\text { porque estoy pensando en otras cosas. }\end{array}$ \\
\hline Aprovechamiento & 77 & $\begin{array}{l}\text { Frecuentemente me percato de que no dedico mucho tiempo a esta } \\
\text { asignatura debido a otras actividades. }\end{array}$ \\
\hline
\end{tabular}

Fuente: Elaboración propia.

\section{Conclusiones}

Una vez analizada la muestra, respecto a los objetivos marcados, se ha comprobado que después del análisis estadístico previamente presentado, el uso que los alumnos hacen de los diferentes factores motivacionales en orden decreciente es: metas intrínsecas $(6,07)$, valor de la tarea $(5,91)$, autoeficacia $(5,57)$, metas extrínsecas $(5,39)$, creencias de control $(5,27)$ y ansiedad $(3,11)$. En la orientación de metas intrínsecas, un alto nivel de satisfacción en este componente hace referencia a que la participación del estudiante en ella es un fin en si mismo, más que un medio para alcanzar un determinado fin. Buscando razones como el reto, la curiosidad y el dominio de la tarea. Con el valor de la tarea el 
alumno expresa la importancia, interés y utilidad de la asignatura, si éste la considera importante para la propia formación.

De todas las estrategias de aprendizaje que mide el cuestionario utilizado, las que han hecho uso los estudiantes han sido: elaboración (5,30), organización (5,17), metacognición $(5,06)$, ayuda $(4,68)$, concentración $(4,51)$ y autointerrogación $(4,45)$. Con la elaboración se nos muestra cómo el alumno aplica conocimientos anteriores a través de la información que ya poseía con la nueva para dar respuesta a los problemas que se le presenta. La organización enfatiza en cómo el alumno lleva a cabo el aprendizaje de la información, utilizando para ello esquemas, resúmenes, subrayado, etc. Otras investigaciones (Gargallo et al., 2012; Gargallo y Suárez, 2014) muestran como los alumnos catalogados como excelentes utilizaron mejores estrategias de aprendizaje. El rendimiento académico está afectado por el tipo de atribuciones, el autoconcepto académico y las metas de logro, mientras que las estrategias de aprendizaje estaban determinadas por las metas de aprendizaje (González et al., 1999).

Los ítems con mayor repercusión en la escala motivacional pertenecen a los factores de metas extrínsecas, metas intrínsecas, rendimiento y creencias de control y autoeficacia para el aprendizaje. Una alta involucración por parte del estudiante en la tarea favorece la consecución de los objetivos académicos. Seleccionar las asignaturas que le permitan formarse como docente hace que el nivel de satisfacción sea mayor ya que alcanza las expectativas formadas en el comienzo del curso académico. La creencia del estudiante sobre su propia capacidad para alcanzar un buen rendimiento académico es un correcto punto de partida para aprender los conceptos básicos de la asignatura. Que el alumno relacione el dominio de la materia de la asignatura con su esfuerzo y modo de estudio muestra la capacidad de control de la asignatura en su autoeficacia para el aprendizaje. Los esfuerzos de los docentes y los recursos materiales invertidos por las facultades, son muy valorados por los alumnos (Cecilia y Ruth, 2014). El estilo cognitivo de los estudiantes y la capacidad de regulación de su aprendizaje están relacionados con el desempeño académico (López, Hederich y Camargo, 2011).

Los ítems que han obtenido una mayor repercusión, y a su vez, una mayor puntuación por parte de los estudiantes en las estrategias de aprendizaje se engloban en los factores de constancia, aprovechamiento, metacognición, organización y elaboración. La asistencia a clase favorece que se lleven al día las actividades y trabajos de cada asignatura para alcanzar las metas propuestas por el profesor. Lo que demanda del alumno diligencia y esfuerzo. Disponer de un lugar fijo para el estudio favorece tanto el aprovechamiento del tiempo como la capacidad para concentrarse en la tarea. Las estrategias que emplea el alumno para entender los conceptos de las asignaturas, los aspectos organizativos son muy valorados como hábitos de estudio. Consiguiendo que el alumno dentro de las estrategias de aprendizaje sea capaz de relacionar los conceptos nuevos con los aprendizajes previo que dispone.

El aprendizaje que llevan a cabo los estudiantes, denominado aprendizaje autónomo, autodirigido, independiente, etc., les permiten analizar los diferentes elementos implicado en el aprendizaje exitoso. Es quizá el que ofrece una mayor aproximación a la comprensión de los procesos de aprendizaje (Valle et al., 2010). Este aprendizaje está relacionado con la motivación, las metas que se marcan y las emociones (Schunk y Zimmerman, 2008). El grado de creatividad del discurso del docente influirá en la motivación del alumno para atraer su atención, establecer formas de relación en el grupo 
clase, etc. (Soler, 2012). Estas metas previamente marcadas dirigen nuestros esfuerzos con el fin de satisfacer una necesidad. Por tanto, el alumnado debe sentirse competente, que posee el control sobre las acciones, autónomo, estar involucrado, etc. y respecto a la tarea que lleva a cabo, debe considerarla satisfactoria, de interés, con posibilidades reales de dominarla y con capacidad de obtener un buen rendimiento. Este rendimiento académico está formado por un conjunto de factores que actúan sobre la persona que está aprendiendo, siendo definido con un valor atribuible al logro que este obtiene de las tareas académicas (Pérez, Ramón y Sánchez, 2000). Las calificaciones que los estudiantes obtienen son un indicador que certifica el logro alcanzado, valorando su rendimiento académico, siempre que se asuma que las notas son el reflejo de los logros académico en los diferentes aprendizajes (Rodríguez, Fita y Torrado, 2004). No hay un criterio consensuado en torno a la calidad de la educación superior, intervienen múltiples factores, el rendimiento académico es una dimensión fundamental. Siendo este la suma de diferentes factores que afectan al estudiante, interacciones de determinantes personales, sociales e institucionales (Garbanzo, 2007).

Desde los diferentes centros de educación debemos ser capaces de trasmitir a los estudiantes las estrategias de aprendizaje necesarias para que alcancen una motivación en el aprendizaje que le permita satisfacer sus necesidades de conocimiento. Todo ello debemos acompañarlo de unas correctas técnicas de estudio por parte de los estudiantes y un método de enseñanza y evaluación acorde por parte de los docentes. Sin olvidar que los progenitores pueden influir en la tasa de rendimiento de sus hijos. Aquellas alumnas cuyas madres poseen estudios medios usan en mayor medida estrategias de memorización en relación a aquellas madres que tienen estudios básicos (Da Cuña et al., 2014).

\section{Referencias}

Amstrong, T. (1991). Awakening your chid's natural genius. Nueva York: The Burning Bush.

Aznar, P. (1992). Constructivismo y educación. Valencia: Tirant lo Blanch.

Ballesteros, S. (1997). Psicología general. Un enfoque cognitivo. Madrid: Universitas.

Bara, P.M. (2001). Estrategias metacognitivas y de aprendizaje: estudio empírico sobre el efecto de la aplicación de un programa metacognitivo, y el dominio de las estrategias de aprendizaje en estudiantes de ESO, BUP y Universidad. Tesis Doctoral. Universidad Complutense de Madrid.

Bernad, J.A. (2000). Modelo cognitivo de evaluación educativa: escala de estrategias de aprendizaje contextualizado. Madrid: Narcea.

Bloom, B. (1976). Manual de evaluación formativa del currículo. Bogotá: Continental Gráfica.

Bruner, J. (1991). Actos de significado. Más allá de la revolución cognitiva. Madrid: Alianza Editorial.

Cánovas, P. y Rius, M. (1993). Proceso de desarrollo y proceso educativo. En P.R. Garfella (Coord.), Construcción humana y procesos de estructuración: propuestas de intervención pedagógica (pp. 45-76). Valencia: Quiles Artes Gráficas.

Cardozo, A. (2008). Motivación, aprendizaje y rendimiento académico en estudiantes del primer año universitario. Laurus, Revista de Educación, 14(28), 209-237.

Carretero, M. (1993). Constructivismo y educación. Zaragoza: Luis Vives. 
Carretero, M. (2001). Introducción a la psicología cognitiva. Buenos Aires: Aique.

Cecilia, M. y Ruth, E. (2014). La evaluación de la calidad de servicio universitario desde la percepción de estudiantes y docentes: estudio de caso. REICE. Revista Iberoamericana sobre Calidad, Eficacia y Cambio en Educación, 12(2), 63-84.

Chipman, S.F. y Segal, J.W. (1985). Higher cognitive goals for education: An introduction. En J.W. Segal, S.F. Chipman y R. Glaser (Eds.), Thinking and learning skills (pp. 1-19). Hillsdale, NJ: LEA.

Da Cuña, I., Gutiérrez, M., Berón, F.J. y Labajos, M.T. (2014). Influencia del nivel educativo de los padres en el rendimiento académico, las estrategias de aprendizaje y los estilos de aprendizaje, desde la perspectiva de género. Journal of Learning Styles, 7(13), 64-84. doi: 10.4321/s2014-98322013000100005

De Vega, M. (1989). Introducción a la psicología cognitiva. Madrid: Alianza Psicológica.

Delclaux, I. (1982). Psicología cognitiva y procesamiento de la información: teoría, investigación y aplicaciones. Madrid: Pirámide.

Eccles, J., Adler T.F., Futterman, R., Goff, S.B., Kaczala, C.M. (1983). Ex-pectancies, values, and academic behaviors. En J.T. Spence (Ed.), Achievement and achievement motivation (pp. 75146). San Francisco, CA: Freeman.

Gagné, E.D. (1991). La psicología del aprendizaje escolar. Madrid: Aprendizaje-Visor.

Gallego, D.J. (2008). Padres y estilos de aprendizaje de sus hijos. Revista Estilos de Aprendizaje, I(1), 4-16.

Garbanzo, G.M. (2007). Factores asociados al rendimiento académico en estudiantes universitarios, una reflexión desde la calidad de la educación superior pública. Revista Educación, 31(1), 43-63. doi: 10.15517/revedu.v31i1.1252

Garfella, P. y Gargallo, B. (1993). La teoría de la equilibración de Piaget: bases para una tecnología de intervención. En P.R. Garfella (Coord.), Construcción humana y procesos de estructuración: propuestas de intervención pedagógica (pp. 89-123). Valencia: Quiles Artes Gráficas.

Gargallo, B. (2012). Estrategias de aprendizaje en estudiantes universitarios excelentes y medios. Su evolución a lo largo del primer año de carrera. RELIEVE, 18(2), 1-22. doi: 10.7203 /relieve.18.2.2000

Gargallo, B. y Suárez, J. (2014). Aproximación al perfil de estudiantes universitarios excelentes. Revista de Docencia Universitaria, 12(2), 143-165.

Gómez, C. y Gargallo, B. (1993). Las bases de una concepción constructivista de la educación. Implicaciones pedagógicas. En P.R. Garfella (Coord.), Construcción humana y procesos de estructuración: propuestas de intervención pedagógica (pp. 35-65). Valencia: Quiles Artes Gráficas.

González, M.C. y Tourón, J. (1992). Autoconcepto y rendimiento académico. Sus implicaciones en la motivación y en la autorregulación del aprendizaje. Pamplona: EUNSA.

González, R. (1999). Un modelo integrador explicativo de las relaciones entre metas académicas, estrategias de aprendizaje y rendimiento académico. Revista de Investigación Educativa, $17(1), 47-70$.

González-Pienda, J.L., González, R., Núñez, J.C y Valle, A. (2002). Manual de psicología de la educación. Madrid: Pirámide.

Hernández Rojas, G. (1998). Paradigmas en psicología de la educación. Barcelona: Paidós. 
Lachman, R., Lachman, J. y Butterfield, E.C. (1979). Cognitive psychology and information processing: an introduction. Tallahassee, FL: CRC Press LLC.

López, O., Hederich, C. y Camargo, A. (2011). Estilo cognitivo y logro académico. Educación y Educadores, 14(1), 67-82. doi: 10.5294/edu.2011.14.1.4

Martínez, C., Rúa, A., Redondo, R., Fabra, M., Núñez, A. y Martín, M. (2010). Influencia del nivel educativo de los padres en el rendimiento académico de los estudiantes de ADE. Un enfoque de género. AEDE, 5(1), 1273-1294.

Monereo, C. (2000). Estrategias de aprendizaje. Madrid: Aprendizaje-Visor.

Navaridas, F. (2002). La evaluación del aprendizaje y su influencia en el comportamiento estratégico del estudiante universitario. Contextos Educativos, 5, 141-156. doi: $10.18172 /$ con.509

Parrales, S. y Solórzano, J. (2014). Motivación y estrategias de aprendizaje del estudiantado de la escuela de orientación y educación especial. Revista Electrónica Actualidades Investigativas en Educación, 14(1), 1-20. doi: 10.15517/aie.v14i1.13201

Pérez-Luño, A., Ramón Jerónimo, J., Sánchez Vázquez, J. (2000). Análisis exploratorio de las variables que condicionan el rendimiento académico. Sevilla: Universidad Pablo de Olavide.

Piaget, J. (1978). La equilibración de las estructuras cognitivas. México: Siglo XXI.

Pintrich, P.R. (1988). A process-oriented view' of student motivation and cognition. En J. Stark y L. Mete (Eds.), Improving teaching and learning through research (pp. 65-79). San Francisco, CA: Jossey Bass.

Pintrich, P.R., DeGroot, E. (1990). Motivational and self-regulated learning components of classroom academic performance. Journal of Educational Psychology, 82(1), 33-40. doi: $10.1037 / 0022-0663.82 .1 .33$

Pintrich, P., Smith, D., García, T. y McKeachie, W. (1991). A manual for of the motivated strategies learning questionnaire (MSLQ). Lansing, MI: The University of Michigan.

Pozo, J.I. (1989). Adquisición de estrategias de aprendizaje. Cuadernos de Pedagogía, 175, 8-11.

Roces, C., Tourón, J. y González, M.C. (1995). Motivación, estrategias de aprendizaje y rendimiento de los alumnos universitarios. Bordón, 47(1), 107-120.

Rodríguez, S., Fita, S. y Torrado, M. (2004). El rendimiento académico en la transición secundaria-universidad. Revista de Educación, 334, 391-414.

Rodríguez Neira, T. (1999). Teorías y modelos de enseñanza. Posibilidades y límites. Lleida: Milenio.

Sampascual, G. (2001). Psicología de la Educación. Madrid: UNED.

Shulman, L.S. (1986). Those who understand: knowledge growth in teaching. Educational Researcher, 15(2), 4-14. doi: 10.3102/0013189x015002004

Schunk, D. y Zimmerman, B. (2008). Motivation and self-regu-lated learning: theory, research and applications. Nueva York: Lawrence Erlbaum.

Soler, J. y Alonso, V. (1996). Estrategias de aprendizaje humano. Valencia: Promolibro.

Soler, R. (2012). ¿Es el lenguaje de los docentes creativo? Análisis de sus expresiones más frecuentes. REICE. Revista Iberoamericana sobre Calidad, Eficacia y Cambio en Educación, $10(3), 88-104$.

Valle, A. (2010). Motivación y aprendizaje autoregulado. Revista Interamericana de Psicología, 44(1), 86-97. 